\title{
Seasonal decline in provisioning effort and nestling mass of Blue Tits Cyanistes caeruleus: experimental support for the parent quality hypothesis
}

\author{
VICENTE GARCÍA-NAVAS ${ }^{1 *}$ \& JUAN JOSÉ SANZ ${ }^{1,2}$ \\ ${ }^{1}$ Área de Zoología, Departamento de Ciencias Ambientales, Facultad de Medio Ambiente, Universidad de \\ Castilla-La Mancha, Avenida Carlos III s/n, 45071 Toledo, Spain \\ ${ }^{2}$ Departamento de Ecología Evolutiva, Museo Nacional de Ciencias Naturales-CSIC, Calle José Gutiérrez \\ Abascal 2, 28006 Madrid, Spain
}

\begin{abstract}
A common feature of many birds breeding in seasonal environments is that fitnessrelated parameters such as nestling mass or survival decline as the breeding season progresses. Consequently, there is a tendency for early breeders to have better reproductive performance than individuals breeding later in the season. This variation could be caused by factors associated with the date of laying, such as changing environmental conditions (date hypothesis), or by differences in parental quality between early and late breeders (parent quality hypothesis). To evaluate the relative importance of both hypotheses, we manipulated hatch dates of Blue Tits Cyanistes caeruleus by exchanging clutches with different incubation stages and assessed the impact on nestling mass, nestling diet and provisioning rates. Mean nestling mass declined significantly over the season. This was the combined result of differences in parental quality, which dominated in the early part of the season, and the influence of hatching date (date effect per se), which prevailed later in the season. Nestling diet composition was apparently uninfluenced by the manipulation, suggesting that deteriorating food supplies are the primary reason for the seasonal variation in the nestling diet. Counter to the date hypothesis, delayed parents did not feed their young less than control pairs did, but in fact exhibited higher provisioning rates. Our results suggest that in this population, parental quality seems to constrain post-hatching reproductive performance and such intrinsic limitations may help to explain why certain individuals breed later.
\end{abstract}

Keywords: date hypothesis, hatch date, Mediterranean, provisioning effort, timing of breeding.

A seasonal decline in reproductive performance is a common life-history trait among birds reproducing in seasonal environments, where the breeding period is a more or less fixed window in the annual cycle. Two mechanisms are commonly proposed to explain why early breeders are generally more successful than late breeders: the date hypothesis and the parent quality hypothesis (Verhulst \& Nilsson 2008). The date hypothesis holds that seasonal changes in the environment (e.g. deteriorating food supplies) are the primary cause of a seasonal

*Corresponding author.

E-mail: vicente.garcianavas@uclm.es decline in breeding performance (e.g. Brinkhof et al. 1993, Norris 1993, Lepage et al. 1999). Under the parent quality hypothesis, seasonal variation in reproductive performance is due to differences in phenotype (e.g. age, condition or provisioning abilities; De Forest \& Gaston 1996, Hipfner 1997, Nisbet et al. 1998, de Neve et al. 2004) or territory quality (Alatalo et al. 1986) between early and late breeders.

One type of experiment proposed to test these hypotheses is hatch-date manipulation. Although the hypotheses are not mutually exclusive (e.g. Verhulst et al. 1995), they do yield distinct predictions of the outcome of such experiments. The date hypothesis predicts that the experimentally 
advanced and delayed pairs will follow the annual seasonal trend. The parent quality hypothesis predicts that performance of advanced and delayed pairs will be related to their intended hatching date, not to the actual one; delayed pairs should have the best performance for a given hatch date and advanced pairs the poorest.

In comparing broods with the same hatching date, one would expect that delayed broods would perform worse than controls when breeding performance is linked to a gradual change in the environment that affects all individuals equally. The opposite should be true in the case of pairs experimentally advanced. Under the parent quality hypothesis, breeding performance of experimental pairs should match those of control pairs hatched on the same dates. For more details about predictions derived from these types of studies, see Wardrop and Ydenberg (2003).

Although the fitness consequences of variation in timing of reproduction and the mechanisms underlying seasonal decline in reproductive performance have been assessed by a number of experimental studies (reviewed in Verhulst \& Nilsson 2008), few of these studies have measured parental quality (Hatchwell 1991). In this experimental study of Blue Tits Cyanistes caeruleus, we used feeding frequency as a proxy for parental effort, in order to assess seasonal variation in parental effort and its effects on reproductive parameters. We attempt to control for potential confounding factors such as egg quality (by using eggshell coloration as a proxy) and female body condition (measured as body mass). This is because such factors may introduce biases in cross-fostering experiments and thus lead to unequal opportunities to show evidence in favour of these hypotheses (Wardrop \& Ydenberg 2003, Arnold et al. 2004, Verhulst \& Nilsson 2008).

\section{METHODS}

\section{Study area and fieldwork}

The data for the current study were collected during the breeding season of 2009 (late March to mid-June) in Quintos de Mora (Toledo Province, central Spain, $39^{\circ} 25^{\prime} \mathrm{N}, 4^{\circ} 04^{\prime} \mathrm{W}$ ), an area belonging to the National Wildlife Reserves. The reserve is located in the northeast spurs of Montes de Toledo and comprises a mosaic of forest types, with typical Mediterranean flora as the predominant vegetation. A total of 180 wooden nestboxes, distributed mostly in two adjacent plots dominated by Pyrenean and Zeen Oak (Quercus pyrenaica and Quercus faginea, respectively), were used in this experiment. Nestboxes were checked to record the laying date of the first egg ( $1=1$ st April), clutch size, hatching date, number of young that hatched and number that subsequently fledged. Adults were captured while feeding 8-day-old nestlings, aged (first year breeder or older) based on plumage characteristics, sexed according to the presence or absence of a brood patch, and individually identified with metal rings. Birds were weighed with a portable electronic balance to a precision of $0.1 \mathrm{~g}$, and their tarsus length (to the nearest $0.01 \mathrm{~mm}$ ) was measured using a digital calliper. The same parameters were measured in nestlings when they were 13 days old. On day 20 post-hatching, nests were checked to determine the breeding success number of fledged young/number of eggs hatched) of each pair.

\section{Experimental protocol}

Timing of parental care was manipulated by exchanging clutches laid on different dates and thus differing in the onset of incubation. We selected dyads of clutches of equal size (allowing for one egg difference) that differed by 6 days in expected hatching date (allowing for 1 day difference). As soon as the female of the later clutch began incubation, the clutch was matched and exchanged with another in which incubation had started 6 days ( \pm 1 day) earlier. For each dyad of experimental clutches we selected a third clutch that served as a control. This clutch was of the same size as the two manipulated clutches $( \pm 1$ egg) and their laying date equal ( \pm 1 day) to that of either the advanced or the delayed clutch. The eggs were transported in less than $10 \mathrm{~min}$ in warm insulated boxes filled with cotton. Control clutches were not swapped between nests; we removed the eggs and returned them to the nest after a period of time equivalent to that employed in egg transfer between early and late clutches.

The magnitude of the manipulation (hereafter called hatching difference, i.e. the number of days elapsed between the observed hatching date and the original hatching date) varied from -6 to 0 days (mean $\pm \mathrm{sd}$ : $2.8 \pm 4.2$ ) for advanced pairs and from 2 to 10 days (mean $\pm \mathrm{sd}$ : $6.3 \pm 4.3$ ) for delayed pairs. Negative values represent advanced 
broods and positive values delayed broods. We designated the original hatching date as the expected hatching date prior to egg swapping. Because incubation normally lasts 13 days in this species, the hatching date was calculated accordingly (Sanz \& García-Navas 2009). In all, 36 clutches were manipulated (18 pairs were advanced and 18 pairs delayed) and 18 pairs served as controls. One delayed brood was deserted 8 days after hatching, and one advanced brood was predated by a snake just after hatching.

\section{Speckling assessments}

A potential bias of hatch-date manipulation experiments is the existence of maternal effects acting via the egg. If seasonal variation in reproductive success is due (in part) to a seasonal variation in egg characteristics, such a variable must be controlled to ascertain whether there is indeed a causal relationship between reproductive success and timing of breeding or whether such a relationship is driven largely by a third variable (egg quality) whose association with breeding date has not been altered by the experiment (Verhulst \& Nilsson 2008). In this study we use egg appearance as a proxy for egg quality. Previous studies carried out on this study population revealed that spotting distribution is strongly associated with hatching success (Sanz \& García-Navas 2009) and the genetic quality of the female (García-Navas et al. 2009). To assess this trait, all eggs (340, belonging to 48 nests) were scored by the same observer (V.G.N.) on the basis of the degree of spotting distribution (ranked in 0.5 -point increments, from 1 for eggs whose spots are all on the broad end, to 5 for eggs with speckling evenly distributed over the shell surface; see Gosler et al. 2000) after clutch completion.

\section{Provisioning effort and nestling diet}

Parental provisioning effort and nestling diet were studied by means of video recordings taken when nestlings were 10-11 days old. Handycams (Sony DCR-SR290E; Sony Corp., Tokyo, Japan) were placed inside a wooden enclosure fixed to the back of the nestbox facing the entrance hole in such a way that adults entering were filmed and delivered prey could be identified. To habituate the birds to this device the original nestbox was replaced with the model adapted to the video camera 1 day prior to filming. In our study area all nestboxes are pro- tected from predators (Small-spotted Genet Genetta genetta and Stone Marten Martes foina) with PVC pipes fixed to the hole-entrance, permitting observation of the birds when they go through the nestbox entrance. Feeding activity (unmanipulated clutches $n=12$; control clutches $n=11$; experimental clutches $n=30$ ) was videotaped for 150 min between 08:30 and 11:30 h. The number of feeding events per chick detected within this period was taken as an estimate of parental effort (feeding rates of both pair members are combined) during the brood-rearing phase. The first half-hour of recording was discarded as birds took time (10-20 min) to resume feeding activity after the disturbance that the video camera installation entails. The following $45 \mathrm{~min}$ were examined in detail, as this time interval was also used to study the diet of nestling Blue Tits. Prey items were classified as Lepidoptera larvae (tortricids, geometrids and noctuids) or others (including spiders, pupae, cocoons, moths, Hymenoptera, Hemiptera and vegetable tissue). The proportion of Lepidoptera larvae in the diet was calculated as the number of Lepidoptera larvae brought to the young divided by the total number of provisioning trips.

\section{Data analysis}

By using data for unmanipulated $(n=88)$ and control pairs $(n=18)$, we checked for seasonal variation in breeding parameters (nestling body mass, egg spottiness, provisioning effort and nestling diet) by fitting a model with hatching date as an independent variable. To disentangle the effects of date (timing) and parental quality on these variables, we used generalized linear mixed models and multiple regression models to assess whether breeding traits in experimental clutches deviated from values expected on the basis of the annual seasonal trend by including treatment (unmanipulated, control, advanced, delayed) and its interaction with observed hatch date as factors in the model. In a second step, the existence of differences in the strength of the experimental hatching date effect was assessed by examining the interaction of hatching difference $\times$ observed hatch date. If the effect of the experiment on a certain breeding parameter changed throughout the season, we analysed early and late clutches separately. However, if there was no evidence of contrasting patterns within the season, analyses were 
performed over the entire breeding period. We then ran multiple regression models including (1) hatching date as predictor variable and (2) the terms original hatching date and change in hatching date (i.e. hatching difference) combined to find the most parsimonious model to fit the data. If the date hypothesis were supported, we would expect that removal of the manipulation period (i.e. hatching difference) from the combined model would reduce the predictive power of the model. In addition, a model with only actual hatching date should explain the data as well as a model with original hatching date and manipulation period combined. Under the parental quality hypothesis, manipulation period should increase the goodness of fit of a model including the actual hatching date (see Verboven and Verhulst (1996) for more information on predictions involving hatch-date manipulation experiments).

Statistical analyses were performed with STATISTICA 6.0 (Statsoft Inc., Tulsa, OK, USA) and sPSS v.15 (SPPS Inc., Chicago, IL, USA). ANOVA and Wilcoxon tests were used to compare reproductive parameters among experimental groups using either intended or observed hatching dates. Posthoc pairwise comparisons were conducted using Fisher's test. All proportions were arc-sine square root-transformed prior to entry in the model. Degrees of freedom can differ between analyses because we did not have all the measurements for all the birds. Data are given as the mean \pm sd unless stated otherwise.

\section{RESULTS}

Basic breeding parameters for experimental and control pairs are summarized in Table 1. Comparisons between advanced, delayed and control groups revealed that there were no differences in clutch size, egg spottiness or female age. Neither breeding success (number of fledged young) nor hatching success (number of eggs hatched) varied significantly among experimental groups.

When exploring the natural relationship between breeding parameters and hatching date on unmanipulated and control clutches, no relationship was found between either egg spottiness (measured as the distribution of speckles across the egg's surface) or breeding success (proportion of hatched young fledging) and calendar date (Table 2). This suggests that neither variable was a potential bias for comparisons between advanced and delayed clutches, and hence neither was considered in subsequent analyses.

\section{Nestling mass}

Nestling body mass in unmanipulated and control clutches gradually declined with progress of the breeding season (Table 2). There was a significant effect of experimental treatment when data from manipulated clutches were added $\left(F_{2,103}=5.71\right.$, $P<0.01)$ but this effect did not vary with hatch date (treatment $\times$ hatch date: $F_{2,101}=4.27$, $P=0.14$ ). However, when hatch-date manipula-

Table 1. Breeding parameters for advanced, control and delayed Blue Tit groups.

\begin{tabular}{|c|c|c|c|c|c|c|}
\hline & \multicolumn{3}{|c|}{ Treatment } & \multirow[b]{2}{*}{ df } & \multirow[b]{2}{*}{$F$} & \multirow[b]{2}{*}{$P$} \\
\hline & Advanced & Control & Delayed & & & \\
\hline Clutch size & $7.00 \pm 0.31(18)$ & $7.22 \pm 0.31(18)$ & $7.05 \pm 0.31(18)$ & 2.51 & 0.13 & 0.87 \\
\hline Egg spottiness ${ }^{1}$ & $3.55 \pm 0.13(15)$ & $3.61 \pm 0.12(17)$ & $3.54 \pm 0.13(16)$ & 2.45 & 0.11 & 0.89 \\
\hline Hatching success & $89.35 \pm 4.28(17)$ & $85.10 \pm 4.16(18)$ & $79.43 \pm 4.16(18)$ & 2.50 & 1.39 & 0.26 \\
\hline Male age & $0.73 \pm 0.10(15)$ & $0.86 \pm 0.10(14)$ & $0.92 \pm 0.11(12)$ & 2.38 & 0.82 & 0.45 \\
\hline Female age & $0.44 \pm 0.12(16)$ & $0.57 \pm 0.12(16)$ & $0.64 \pm 0.12(17)$ & 2.46 & 1.34 & 0.27 \\
\hline Female mass & $9.67 \pm 0.11^{a}(15)$ & $9.62 \pm 0.11^{a}(17)$ & $9.32 \pm 0.11^{\mathrm{b}}(15)$ & 2.44 & 1.79 & 0.07 \\
\hline Feeding frequency ${ }^{2}$ & $10.30 \pm 1.45^{\mathrm{a}}(14)$ & $11.17 \pm 1.64^{\mathrm{a}}(11)$ & $15.21 \pm 1.36^{\mathrm{b}}(16)$ & 2.38 & 3.44 & 0.04 \\
\hline Nestling mass $(\mathrm{I})$ & $9.63 \pm 0.16^{a}(9)$ & $9.91 \pm 0.16^{\mathrm{a}}(9)$ & $10.40 \pm 0.16^{\mathrm{b}}(9)$ & 2.24 & 5.58 & 0.01 \\
\hline Nestling mass (II) & $9.65 \pm 0.17(8)$ & $9.84 \pm 0.16(9)$ & $9.80 \pm 0.17(8)$ & 2.22 & 0.09 & 0.68 \\
\hline$\%$ Lepidoptera $^{3}$ & $64.59 \pm 4.37(14)$ & $61.50 \pm 4.93(11)$ & $58.54 \pm 3.87(16)$ & 2.38 & 0.57 & 0.57 \\
\hline Number of fledged young & $6.17 \pm 0.43(17)$ & $6.11 \pm 0.41(18)$ & $5.59 \pm 0.43(17)$ & 2.49 & 0.56 & 0.57 \\
\hline
\end{tabular}

Sample sizes are given in parentheses. Means \pm se followed by different lower-case letters are statistically significant $(P<0.05)$. Sample sizes are given in parentheses.

${ }^{1}$ Degree of clumping of speckles across the egg's surface. ${ }^{2}$ Number of feeding trips per chick made by parents to their young during a 150-min period. ${ }^{3}$ Proportion of caterpillars in nestling diet. (I) Early season. (II) Late season. 
Table 2. Multiple regression models for several breeding parameters in clutches that were not subjected to treatment (control $n=18$ and unmanipulated broods $n=88$ ).

\begin{tabular}{|c|c|c|c|c|c|}
\hline & $\begin{array}{l}\text { Nestling body } \\
\text { mass }\end{array}$ & $\begin{array}{c}\text { Egg } \\
\text { spottiness }\end{array}$ & $\begin{array}{l}\text { Feeding } \\
\text { frequency }\end{array}$ & $\begin{array}{l}\text { Percentage } \\
\text { Lepidoptera }\end{array}$ & $\begin{array}{l}\text { Breeding } \\
\text { success }\end{array}$ \\
\hline Nestbox plot & $0.22 \pm 0.06^{\star * *}$ & NS & NS & $8.42 \pm 2.87^{*}$ & NS \\
\hline Hatch date & $-0.02 \pm 0.01^{\star *}$ & NS & $-2.46 \pm 0.32^{*}$ & $-1.53 \pm 0.63^{\star *}$ & NS \\
\hline Hatch date $^{2}$ & NS & NS & NS & NS & NS \\
\hline Clutch/brood size & $-0.11 \pm 0.02^{\star * *}$ & NS & $10.87 \pm 1.21^{* \star *}$ & NS & NS \\
\hline Nestling size & $0.38 \pm 0.12^{\star \star \star *}$ & - & - & - & - \\
\hline Constant & $-17.06 \pm 6.53$ & - & $117.32 \pm 14.32$ & $126.14 \pm 26.33$ & - \\
\hline \multicolumn{6}{|l|}{ Model } \\
\hline$R^{2}$ & 0.44 & - & 0.43 & 0.29 & - \\
\hline$F$ & 13.35 & - & 7.93 & 4.32 & - \\
\hline df & 4,69 & $n=48$ & 2,20 & 2,20 & $n=80$ \\
\hline
\end{tabular}

The models only include the significant terms whose coefficients appear in the Table. See text for more details.

NS, $P>0.05 ;{ }^{*} P<0.05 ;{ }^{\star \star} P<0.01 ;{ }^{* \star \star} P<0.001$.

Table 3. Multiple regression models for nestling body mass, female body mass, feeding frequency and proportion of Lepidoptera larvae in nestling diet including (a) observed hatching date, and (b) original hatching date in combination with the magnitude of the manipulation (hatching difference) as independent variables.

\begin{tabular}{|c|c|c|c|c|c|c|}
\hline & & \multicolumn{2}{|c|}{ Nestling body mass } & \multirow{2}{*}{$\begin{array}{c}\text { Female body } \\
\text { mass }\end{array}$} & \multirow[b]{2}{*}{ Feeding frequency } & \multirow{2}{*}{$\begin{array}{l}\text { Percentage } \\
\text { Lepidoptera }\end{array}$} \\
\hline & & Early season & Late season & & & \\
\hline \multirow[t]{3}{*}{ (a) Model I } & $R^{2}$ & 0.01 & 0.26 & 0.05 & $<0.01$ & 0.53 \\
\hline & $F$ & 1.17 & 9.08 & 3.33 & 0.19 & 46.21 \\
\hline & df & 1,25 & 1,22 & 1,44 & 1,39 & 1,39 \\
\hline Hatch date & & $0.03 \pm 0.02(\mathrm{NS})$ & $-0.05 \pm 0.02^{\star *}$ & $-0.02 \pm 0.01$ (NS) & $-0.06 \pm 0.14(\mathrm{NS})$ & $-1.73 \pm 0.25^{\star \star}$ \\
\hline Constant & & $9.13 \pm 0.79$ & $12.04 \pm 0.75$ & $10.17 \pm 0.34$ & $14.60 \pm 4.94$ & $120.74 \pm 8.89$ \\
\hline \multirow[t]{3}{*}{ (b) Model II } & $R^{2}$ & 0.30 & 0.24 & 0.11 & 0.17 & 0.53 \\
\hline & $F$ & 6.71 & 4,69 & 3.69 & 5.25 & 23.89 \\
\hline & df & 2,24 & 2,21 & 2,43 & 2,38 & 2,38 \\
\hline Original hatch date & & $-0.01 \pm 0.01$ (NS) & $-0.06 \pm 0.02^{\star \star}$ & $-0.01 \pm 0.01$ (NS) & $-0.13 \pm 0.13(\mathrm{NS})$ & $-1.78 \pm 0.26^{\star \star \star}$ \\
\hline Hatching difference & & $0.07 \pm 0.02^{\star \star}$ & $-0.04 \pm 0.02(\mathrm{~d})$ & $-0.04 \pm 0.01^{*}$ & $0.46 \pm 0.21^{*}$ & $-1.37 \pm 0.41^{\star \star}$ \\
\hline Constant & & $9.92 \pm 0.70$ & $12.19 \pm 0.79$ & $10.12 \pm 0.33$ & $15.74 \pm 4.45$ & $121.54 \pm 8.88$ \\
\hline
\end{tabular}

Coefficients and the statistics ( $P$-values, in parentheses) are given. NS, $P>0.1$, a $P<0.1,{ }^{*} P<0.05 ;{ }^{* \star} P<0.01 ;{ }^{* \star *} P<0.001$.

tion was expressed as a continuous variable (days of delay or advance), then its effect on nestling mass did vary with season (hatching difference $x$ hatch date interaction: $F_{1,47}=7.17, \quad P=0.01$ ). Specifically, early in the breeding season (date range 18-33), nestling mass was positively correlated with the change in hatching date, and delayed pairs raised heavier nestlings than control pairs (Table 1). Addition of hatching difference to a model with actual hatching date as a predictor variable (Table $3 \mathrm{a}$ ) reduced the deviance, but not significantly $(\Delta \mathrm{D}=2.62, \quad P=0.1)$ probably due to the small sample size. On the other hand, multiple regression analysis showed that the model with original hatching date and hatching difference as predictor variables (Table $3 b$ ) produced a better fit than that based only on the observed hatching date (Table 3a). Later in the breeding season (date range 34-50), mean nestling mass did not differ significantly among treatments (Table 1), or with hatch-date manipulation (Fig. 1). Accordingly, addition of hatching difference to a model with original hatching date as a predictor variable reduced the deviance non-significantly $(\Delta \mathrm{D}=0.08, \quad P>0.5)$. This combined model (Table $3 \mathrm{~b}$ ) also had a similar predictive power to the model based on the observed hatching date (Table 3a). 


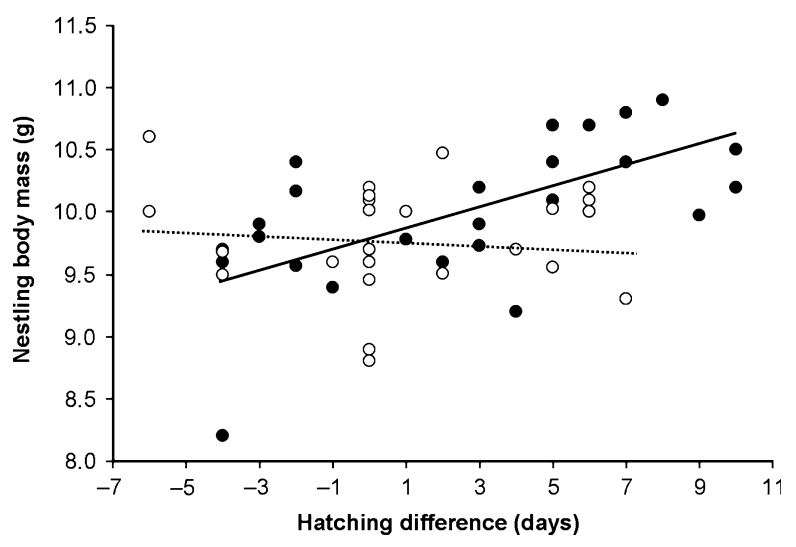

Figure 1. Relationship between nestling body mass and the magnitude of the manipulation (hatching difference) in the early (solid line and filled circles) and late (broken line and empty circles) part of the season. Mean nestling mass is associated positively with hatching difference early in the season $\left(F_{1,25}=13.92, P<0.001\right.$, coefficient: $\left.0.07 \pm 0.02, R^{2}=0.33\right)$ but not late in the season $\left(F_{1,22}=0.04, P=0.83\right.$, coefficient: $-0.01 \pm 0.01, R^{2}=0.01$ ). The season was divided into early and late at the median date. Negative values of hatching difference represent advanced broods and positive values represent delayed broods.

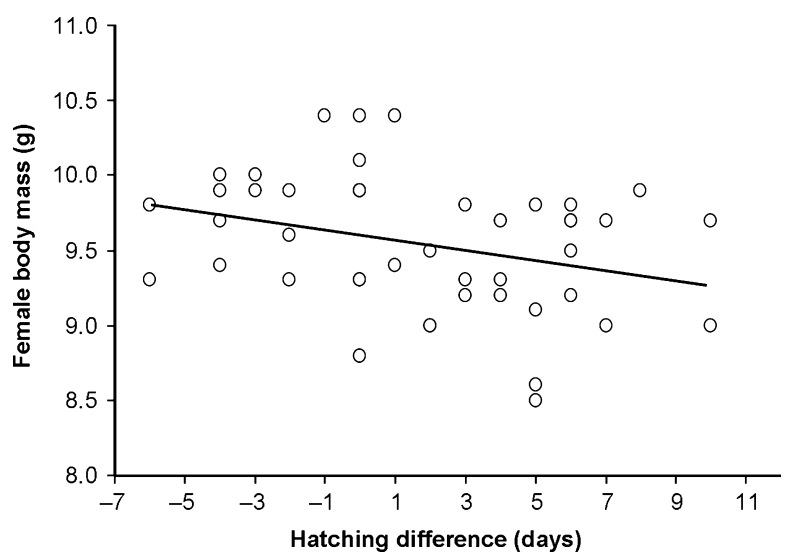

Figure 2. Female body mass during nesting in relation to the number of days elapsed between the intended and the observed hatch date (hatching difference; $F_{1,44}=4.82$, $P=0.03$, coefficient: $-0.03 \pm 0.02, R^{2}=0.08$ ). Negative values of hatching difference represent advanced broods, and positive values represent delayed broods.

\section{Female mass}

Female body mass increased through the season in unmanipulated and control broods (unmanipulated $n=52$, control $n=17 ; \quad F_{1,67}=5.84, \quad P=0.03$ ). However, female body mass was negatively correlated with hatching difference in manipulated clutches (Fig. 2), i.e. the condition of females whose incubation period was prolonged (delayed clutches) was poorer than that of females whose incubation period was shortened (advanced clutches). This implies that increasing incubation time may have negative effects on female condition (body mass).

\section{Provisioning effort}

The number of feeding events by parents declined significantly over the course of the breeding period in unmanipulated broods (Table 2). When experimental broods were included in the model, there was a strong treatment effect and marginal effect of the treatment $\times$ hatch date interaction (treatment: $F_{2,49}=17.33, P<0.001$, treatment $\times$ hatch date: $F_{2,49}=3.68, P=0.06$ ), which indicates that feeding effort differed between treatments. Specifically, feeding frequencies were not significantly different between advanced and control pairs but delayed pairs fed their young more than control pairs did (Table 1). However, variation in the seasonal trend between treatments was only slightly different from that expected from the natural seasonal trend. When hatch-date manipulation was expressed as a continuous variable (days of delay or advance), its effect on feeding frequency did not vary with season (hatching difference $x$ hatch date interaction: $F_{1,37}=1.51, P=0.23$ ), and thus early and late clutches could be pooled. The multiple regression analysis showed that feeding frequency was linearly and positively correlated with the change in hatching date as predicted by the parent quality hypothesis (Fig. 3). The inclusion of hatching difference in a model with actual hatching date as a predictor variable decreased the deviance significantly $(\Delta \mathrm{D}=6.75, P<0.01$, Table 3a,b).

\section{Nestling diet composition}

The proportion of Lepidoptera larvae in nestling diet among control and unmanipulated pairs declined significantly with hatching date (Table 2). When manipulated clutches were added to the model, there was no treatment effect or seasonal interaction (treatment: $F_{2,58}=1.83, \quad P=0.17$, treatment $\times$ hatch date: $F_{2,48}=2.13, \quad P=0.13$ ). Equally, there was no evidence that seasonal variation in the proportion of caterpillars in nestling diet varied between treatments when manipulation was expressed as a continuous variable (hatching 


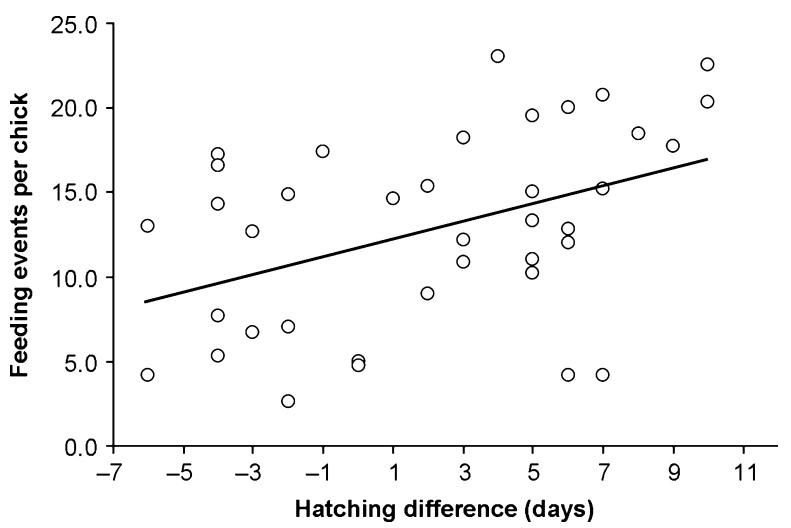

Figure 3. Feeding frequency (number of visits per chick) in relation to the number of days elapsed between the intended and the observed hatch date (hatching difference; $F_{1,39}=9.50$, $P<0.01$, coefficient: $0.56 \pm 0.18, R^{2}=0.17$ ). Negative values of hatching difference represent advanced broods and positive values represent delayed broods.

difference $\times$ original hatch date interaction: $F_{1,37}=0.57, P=0.22$ ). Accordingly, the multiple regression analyses showed that variation in the type of prey devoted to young is best explained by actual hatching date. The model with only observed hatching date (Table 3a) explained the data as well as the model with original hatching date and hatching difference combined (Table 3b). The addition of hatching difference to a model with original hatching date as an independent variable did not result in a significant change in the deviance $(\Delta \mathrm{D}=3.34, P=0.1)$. This indicates that diet composition varied according to the seasonal trend, and thus quality differences between individuals had no additional effect beyond the effect of breeding time itself.

\section{DISCUSSION}

Mean nestling mass declined with advance of the breeding season. In the first half of the season but not the second, there was an additional parental or territory quality effect on nestling body mass. Thus, nestling mass variation did not respond to experimental manipulation uniformly over the course of the breeding period. The relative importance of these factors (parental or territory quality, timing of breeding) could vary in the short term, supporting both hypotheses within the same season. This concurs with Brinkhof (1997), who found that body size of European Coot Fulica atra chicks was associated with the original hatching date in the first part of the season (parent quality hypothesis), but with the assigned hatching date rather than the original hatching date in the second half (date hypothesis). Wardrop and Ydenberg (2003) also found support for both hypotheses at different points in the season when exploring seasonal variation in nestling mass in Tree Swallows Tachycineta bicolor, but in this case found evidence for the date hypothesis early in the season, and the parent quality hypothesis later. What factors influencing reproductive performance are responsible for the differences observed between the two halves of the season in our study? One possible explanation is lower variability in quality among individuals in the late period of the season in comparison with the early period. However, the variability (coefficient of variation) in provisioning effort detected for early breeders was higher with respect to individuals that bred later (early breeders: $48.3 \%$, late breeders: $21.4 \%$ ) so this explanation seems unlikely. Alternatively, territory quality may also play a role in the predominance of parent/territory quality effects in the early period as differences in quality between rearing environments are likely to diminish as the season progresses (Cody 1985). In other words, late in the season the shortage of food may reduce spatial heterogeneity in habitat quality, thus strengthening the influence of date and leading to timing effects being more pronounced (Verhulst \& Tinbergen 1991, Brinkhof 1997). Hence, our results support Wardrop and Ydenberg (2003) when they note that forces influencing reproductive output of early and late breeders may differ.

Among early breeders, the effect that the hatchdate manipulation had on nestling mass was not symmetric; that is, one of the two experimental groups deviated more from the unmanipulated seasonal trend than the other (Table 1). Early-breeding pairs that were experimentally delayed raised significantly heavier nestlings than expected on the basis of the nestling weights of unmanipulated, late breeders, which implies that the delayed pairs may have compensated for less favourable environmental conditions. However, when late-breeding pairs were experimentally advanced, the differences between the mean body mass of nestlings from these pairs and those raised in control or unmanipulated nests were not as marked. Such a tendency is in agreement with previous experimental studies (Herring Gull Larus argentatus Brouwer et al. 1995, Brinkhof 1997, Wardrop \& Ydenberg 2003; 
Common Tern Sterna hirundo Arnold et al. 2004), which found evidence supporting the parent quality hypothesis for delayed clutches and evidence supporting the date hypothesis for advanced clutches. In our study, advancing hatch date did not provide strong evidence in favour of the date hypothesis but rather a more marginal support for the parent quality hypothesis. An explanation for the above effect might be that energy constraints during the early phase of reproduction (egg-laying and incubation period) would outweigh the costs that the chick-rearing period entails (Reid et al. 2000, de Heij 2006). In this regard, the difference in quality between early and late breeders stems from their ability to produce a clutch early in the season (thus facing costs derived from breeding early) and not their ability to rear a brood once it has hatched (Verhulst \& Nilsson 2008). Thus, once this critical stage (i.e. egg-laying and/or incubation period) has passed, late breeders may be as successful as early pairs.

Timing of reproduction cannot be manipulated directly without causing modifications to other aspects of breeding performance. In cross-fostering experiments, timing of parental care is modified by means of inducing birds to incubate their clutch for a shorter (advanced) or longer (delayed) period, thereby potentially reducing or increasing incubation costs, respectively. Here, we provide evidence of a harmful effect of prolonged incubation period on female body mass, which is in agreement with previous studies on Barnacle Geese Branta leucopsis (Tombre \& Erikstad 1996) and Blue Tits (Sanz 1999). On the other hand, in contrast to the findings of Wardrop and Ydenberg (2003), females whose incubation period was shortened apparently did not exhibit a better condition in comparison with those in which the length of the incubation period was not altered. Our data show that despite the influence that the manipulation seemed to have on delayed females, it did not seem to obscure parental quality effects. This may be due to the predominance of mechanisms linked to the rearing conditions over those underlying a seasonal deterioration of the environment. Hence, one might speculate that the detected parental quality effects (differences in phenotypic quality of adults) could have been larger if the reproductive costs (i.e. incubation period) were not manipulated (see also Arnold et al. 2004). It is also plausible that parental quality effects were not masked by this potential bias due to the absence of any correlation between female body mass and parental quality. In this sense, we did not find an association between this trait and female provisioning ability, an indirect measure of parental quality (Spearman's correlation $r_{17}=0.11, P=0.68$ ).

Another potential confounding factor in clutchswapping experiments is the existence of maternal effects acting via the egg. As our experimental design allowed us to manipulate the timing of parental care but not the hatching date of the fostered clutch, this approach is appropriate only when egg quality does not decline with the season. In this study, there was no relationship between eggshell pigmentation pattern (a good indicator of egg quality in the Blue Tit; Sanz \& García-Navas 2009) and calendar date, suggesting that seasonal variation in egg condition may not have been a cause of seasonal variation in nestling body condition.

Provisioning frequency (number of feeding events per chick) was positively correlated with the difference between the original and experimentally induced hatching date, which is consistent with the parent quality hypothesis. Our results indicate that experimentally delayed pairs fed their young at a higher rate than expected for their chosen (original) hatch date. The simplest explanation for this result is that early breeders may be more proficient in finding food or capturing prey than those breeding later. Previous studies (Aebischer 1993, Morbey \& Ydenberg 2000) have found evidence in support of this idea but in none of these was parental quality (as we understand it, see Wilson \& Nussey 2010) measured indirectly.

Alternatively, a shift in the type of prey available to birds would explain the existence of a decline in parental care over time. Mägi et al. (2009) found that Great Tits Parus major reduced their provisioning rates as the season progressed, as adult Lepidoptera (moths) became a more common prey in the late season (see also Barba et al. 1994). In a study with House Sparrows Passer domesticus, Schwagmeyer and Mock (2003) also related the seasonal decline in per-chick deliveries to an increase in the size of prey items (large Lepidoptera larvae and Orthoptera) later in the summer. However, in our study area, neither adult Lepidoptera nor Orthoptera constituted a common prey for Blue Tits in the late season. In our population, pupae of tortricids were the second most frequent prey type, and comprised the bulk of the diet at the end of the season (Garcia-Navas \& 
Sanz, submitted). The nutritional value of this resource is likely to be inferior to that of the caterpillars (even if only due to size differences) and thus it should be expected that late breeders are fed more often to compensate for the small size and low quality of this alternative prey (NaefDaenzer et al. 2000). Therefore, the seasonal decline in provisioning rates found in our study area is unlikely to be related to a switch to the most profitable type of prey for birds at different points of the season.

Another plausible explanation for the observed seasonal decline in provisioning effort of Blue Tits is a shift in the trade-off between current and future reproduction (Drent \& Daan 1980). This shift in life-history trade-offs determining parental effort may be prompted by two deleterious consequences of nesting late: (1) the reduction in the time window for post-breeding activities and (2) the frequently observed decline in the reproductive value of young. In regard to the former, late-breeders may be constrained by necessary preparations for the non-breeding season. One such preparation is post-nuptial moult, which takes place as the breeding season ends (Jenni \& Winkler 1994). The requirement to moult represents a conflict in resource allocation between two energy-demanding processes: reproduction and plumage renewal (e.g. Siikamäki 1998, Sanz 1999). Hence, birds beginning to moult while still feeding young must face costs derived from the production of new feathers and impaired flight efficiency, and as a result their provisioning effort could be reduced (Svensson \& Nilsson 1995 and references therein). However, in the present study only two of 102 individuals were found to be moulting while still feeding young. Alternatively, a reduction in provisioning effort with the advance of the season may be linked to decreased chances of recruitment of late-hatched young (e.g. Barba et al. 1995). Hence, late-breeding parents may voluntarily restrict their provisioning effort if late nestlings are worth less than earlier nestlings (e.g. Curio 1983, Daan et al. 1990). In this case, late breeders would increase their efforts when caring for early-hatched young, and the opposite would be true for early breeders. Such an assumption was confirmed in a chickexchange experiment with Chinstrap Penguins Pygoscelis antarctica, a long-lived species, by Moreno et al. (1997), who found evidence that early breeders invested less effort in late chicks than in early chicks, whereas late-breeders increased investment in early chicks relative to late chicks. However, our data do not support this view. A possible explanation is that the trade-off between current and future reproduction is likely to differ between short-lived (e.g. Blue Tits) and long-lived birds. Reproductive constraints, i.e. incapacity by late-breeders to increase care given to their young, are likely to operate in the first case, and there is less advantage to parents investing in their own future reproductive value at the expense of that of their offspring in short-lived species (Drent \& Daan 1980, Stearns 1992, Moreno 1998).

Nestling diet composition was apparently uninfluenced by the manipulation of the timing of parental care. The seasonal decline in the proportion of Lepidoptera larvae that adult birds provided to their young for manipulated pairs was similar to control pairs. This indicates that deteriorating food supplies are the primary cause of this seasonal variation and that potential differences in quality among early and late breeders do not seem to interact with environmental conditions (territories) at this scale. Thus, our results seem to reveal a much stronger effect of parental quality than territory quality in determining the greater success of early-breeders. However, in the present study we only distinguished between Lepidoptera larvae (caterpillars) and other prey (spiders, pupae, Hemiptera, Orthoptera) and it is likely that within these broad categories, differences in nestling diet could emerge among breeding pairs.

In summary, our study suggests that parental quality seems to constrain post-hatching reproductive performance and, in the same way, that these intrinsic limitations may also help us to explain why certain individuals do not breed earlier. Hence, the possibility that differences in phenotypic quality among early and late breeders may play a more important role than previously proposed in explaining seasonal trends seems plausible, especially in southern Europe where the spread of the breeding season is much greater than in northern latitudes. The fact that parent quality effects tend to be underestimated arises mainly from confounding factors and/or unintentional impacts of the hatch-date manipulation, which could bias the outcome of the experiment towards supporting the date hypothesis. Even in studies in which food was provided to control for environmental deterioration (Brinkhof \& Cavé 1997, Siikamäki 1998) we cannot discard the possible existence of a decrease in parental effort that would be masked by the experimental 
treatment. Thus, the inclusion of measurements of parental effort in studies dealing with fitness consequences of timing of breeding is advisable when considering the reproductive constraints operating not only in the early season but also in the posthatching period.

We are grateful to J. M. Sebastián and C. Rodríguez Vigal (Centro Quintos de Mora, Ministerio de Medio Ambiente) for the facilities offered to work in this restricted area. Jeremy Wilson and two anonymous referees improved the manuscript. V.G.N. was supported by an FPI grant (Ministerio de Ciencia e Innovación-European Social Fund). This study was funded by the project GCL2007-61395 (Ministerio de Ciencia e Innovación).

\section{REFERENCES}

Aebischer, N.J. 1993. Immediate and delayed effects of a gale in late spring on the breeding of the Shag Phalacrocorax aristotelis. Ibis 135: 225-232.

Alatalo, R.V., Lundberg, A. \& Glynn, C. 1986. Female Pied Flycatchers choose territory quality and not male characteristics. Nature 323: 152-153.

Arnold, J.M., Hatch, J.J. \& Nisbet, I.C.T. 2004. Seasonal declines in reproductive success of the Common Tern Sterna hirundo: timing or parental quality? J. Avian Biol. 35: 33-45.

Barba, E., García, D.M., Gil-Delgado, J.A. \& López, G.M. 1994. Moth abundance and breeding success in a Great Tit population where moths are the main nestling food. Ardea 82: 329-334.

Barba, E., Gil-Delgado, J.A. \& Monrós, J.S. 1995. The costs of being late: consequences of delaying great tit Parus major first clutches. J. Anim. Ecol. 64: 642-651.

Brinkhof, M.W.G. 1997. Seasonal decline in body size of Coot chicks. J. Avian Biol. 28: 117-131.

Brinkhof, M.W.G. \& Cavé, A.J. 1997. Food supply and seasonal variation in breeding success: an experiment in the European Coot. Proc. R. Soc. Lond. B 264: 291-296.

Brinkhof, M.W.G., Cavé, A.J., Hage, F.J. \& Verhulst, S. 1993. Timing of reproduction and fledging success in the coot Fulica atra: evidence for a causal relationship. J. Anim. Ecol. 62: 577-587.

Brouwer, A., Spaans, A.L. \& De Wit, A.A.N. 1995. Survival of Herring Gull Larus argentatus chicks: an experimental analysis of the need for early breeding. Ibis 137: 272-278.

Cody, M.L. 1985. Habitat Selection in Birds. Orlando, FL: Academic Press.

Curio, E. 1983. Why do young birds reproduce less well? Ibis 125: 400-404.

Daan, S., Dijkstra, C. \& Tinbergen, J.M. 1990. Family planning in the Kestrel (Falco tinnunculus): the ultimate control of variation in laying date and clutch size. Behaviour 114: 83-116.

De Forest, L.N. \& Gaston, A.J. 1996. The effect of age on timing of breeding and reproductive success in the Thickbilled Murre. Ecology 77: 1501-1511.

Drent, R.H. \& Daan, S. 1980. The prudent parent: energetic adjustments in avian breeding. Ardea 68: 225-252.
García-Navas, V., Ortego, J. \& Sanz, J.J. 2009. Heterozygosity-based assortative mating in Blue Tits (Cyanistes caeruleus): implications for the evolution of mate choice. Proc. R. Soc. Lond. B 276: 2931-2940.

Gosler, A.G., Barnett, P.R. \& Reynolds, S.J. 2000. Inheritance and variation in eggshell patterning in the Great Tit Parus major. Proc. R. Soc. Lond. B 267: 2469-2473.

Hatchwell, B.J. 1991. An experimental study of the effects of timing of breeding on the reproductive success of common guillemots (Uria aalge). J. Anim. Ecol. 60: 721-736.

de Heij, M.E. 2006. Costs of avian incubation: how fitness, energetics and behaviour impinge on the evolution of clutch size. PhD Thesis, University of Groningen.

Hipfner, J.M. 1997. The effects of parental quality and timing of breeding on the growth of nestling Thick-billed Murres. Condor 99: 353-360.

Jenni, L. \& Winkler, R. 1994. Moult and Ageing of European Passerines. London: Academic Press.

Lepage, D., Desrochers, A. \& Gauthier, G. 1999. Seasonal decline of growth and fledging success in Snow Geese Anser caerulescens: an effect of date or parental quality? J. Avian Biol. 30: 72-78.

Mägi, M., Mänd, R., Tamm, H., Sisask, E., Kilgas, P. \& Tilgar, V. 2009. Low reproductive success of Great Tits in the preferred habitat: a role of food availability. Écoscience 16: 145-157.

Morbey, Y.E. \& Ydenberg, R.C. 2000. Seasonal decline in nestling growth: support for the parental-quality hypothesis in Cassin's Auklets. Auk 117: 1065-1068.

Moreno, J. 1998. The determination of seasonal declines in breeding success in seabirds. Etología 6: 17-31.

Moreno, J., Barbosa, A., Potti, J. \& Merino, S. 1997. The effects of hatching date and parental quality on chick growth and creching age in the Chinstrap Penguin (Pygocelis antarctica). Auk 114: 47-54.

de Neve, L., Soler, J.J., Soler, M. \& Pérez-Contreras, T. 2004. Differential maternal investment counteracts for late breeding in Magpies Pica pica: an experimental study. J. Avian Biol. 35: 237-245.

Naef-Daenzer, L., Naef-Daenzer, B. \& Nager, R.G. 2000. Prey selection and foraging performance of breeding great tits Parus major in relation to food availability. J. Avian Biol. 31: 206-214.

Nisbet, I.T., Zingo, J.M. \& Gough, G.A. 1998. Variations in growth of Roseate Tern chicks: II. Early growth as an index of parental quality. Condor 100: 305-315.

Norris, K. 1993. Seasonal variation in the reproductive success of blue tits: an experimental study. J. Anim. Ecol. 62: 287-294.

Reid, J.M., Monaghan, P. \& Ruxton, G.D. 2000. Resource allocation between reproductive phases: the importance of thermal conditions in determining the cost of incubation. Proc. R. Soc. Lond. B 267: 34-41.

Sanz, J.J. 1999. Seasonal variation in reproductive success and post-nuptial moult of Blue Tits in southern Europe: an experimental study. Oecologia 121: 377-382.

Sanz, J.J. \& García-Navas, V. 2009. Eggshell pigmentation pattern in relation to breeding performance of blue tits Cyanistes caeruleus. J. Anim. Ecol. 78: 31-41.

Schwagmeyer, P.L. \& Mock, D.W. 2003. How consistently are good parents good parents? Repeatability of parental 
care in the House Sparrow, Passer domesticus. Ethology 109: 303-313.

Siikamäki, P. 1998. Limitation of reproductive success by food availability and breeding time in Pied Flycatchers. Ecology 79: 1789-1796.

Stearns, S.C. 1992. The Evolution of Life Histories. Oxford: Oxford University Press.

Svensson, E. \& Nilsson, J.-Å. 1995. Food supply, territory quality, and reproductive timing in the Blue Tit (Parus caeruleus). Ecology 76: 1804-1812.

Tombre, I.M. \& Erikstad, K.E. 1996. An experimental study of incubation effort in high-Arctic barnacle geese. J. Anim. Ecol. 65: 325-331.

Verboven, N. \& Verhulst, S. 1996. Seasonal variation in the incidence of double broods: the date hypothesis fits better than the quality hypothesis. J. Anim. Ecol. 65: 264-273.

Verhulst, S. \& Nilsson, J.-Å. 2008. The timing of birds' breeding seasons: a review of experiments that manipulated timing of breeding. Philos. Trans. R. Soc. Lond., B, Biol. Sci. 363: 399-410.
Verhulst, S. \& Tinbergen, J.M. 1991. Experimental evidence for a causal relationship between timing and success of reproduction in the great tit Parus major. J. Anim. Ecol. 60: 269-282.

Verhulst, S., van Balen, J.H. \& Tinbergen, J.M. 1995. Seasonal decline in reproductive success of the Great Tit: variation in time or quality? Ecology 76: 2392-2403.

Wardrop, S.L. \& Ydenberg, R.C. 2003. Date and parental quality effects in the seasonal decline in reproductive performance of the Tree Swallow Tachycineta bicolour. interpreting results in light of potential experimental bias. Ibis 145: 439-447.

Wilson, A.J. \& Nussey, D.H. 2010. What is individual quality? An evolutionary perspective. Trends Ecol. Evol. 25: 207214.

Received 25 January 2010;

revision accepted 13 October 2010

Associate Editor: Ian Hartley. 